\title{
Economic impact of COVID-19 pandemic: case of Bulgaria
}

\author{
Nikolay Sterev ${ }^{1 *}$ \\ ${ }^{1}$ University of National and World Economy - Sofia, Bulgaria
}

\begin{abstract}
The COVID-19 has changing the business and has impacted the national economic system. As a result, not just the international trade has been reduced, but there is a sufficient change of the national and global value chains. Thus, the COVID-19 pandemic has not equally impacted on different countries and sectors. Even though the full lockdown has been applied just for 3 months as the preliminary results shows the Bulgarian economy is moved on the back of the EU economies with decrease of $6 \%$ for the last years and some of the sectors was shrinking with more than $50 \%$ for the last year. The paper analyses the internal change of the Bulgarian economic system and covers analysis of labour and innovation added value to the economic growth in Bulgaria. The analysis opposes the Economic growth before and during the COVID-19 crises. It will be a good starting point to analyse the national economic anti-pandemic measures. The paper aims to analyse what was gone wrong and what was done good insight the Bulgarian economy as result of the COVID-19 pandemic's economy transformation. The analysis is good starting point for post-pandemic development measures that are needed for fast economic recovery in the next few years.
\end{abstract}

\section{Introduction}

From the legal point of view, the problem is just on the beginning to be deeply investigated as the COVID-19 rules were reflected in the adoption of the (Bulgarian) Law on Measures and Actions during the State of Emergency, declared by a decision of the (Bulgarian) National Assembly of March 13, 2020, and of certain acts of Council of Ministers and of the Minister of Health. A significant part of the consequences of the pandemics were settled with the issuance of deliberate orders to the Minister of Health. Normative and non-normative legal acts have effect for a short period of time, but a significant part of their legal consequences is to appear in the future.

Among them are, for example, the health care, labor relations, social security relations, loans and banking, court proceedings and others. The adoption of COVID-preventive measures applies to legal relationships that emerged before the pandemic and that continue to exist after the state of emergency.

The social and economic points of view is also just in beginning to set the pandemic social behavior as the Ministry of Economic published draft 8 pages on COVID-19 effects' analysis:

\footnotetext{
* Corresponding author: sterew@abv.bg
} 
- The COVID 19 pandemic has a strong impact on the economic life and activity of Bulgarians. As a result of the application of strict anti-epidemic measures in the battle against the coronavirus, a part of the people remained unemployed; others, stayed at their jobs, but with reduced working hours and pay; a third part, were forcibly placed on leave (paid and unpaid); a fourth part, stayed to work, but worked from home; a fifth part, continued to work on the front line, despite the direct danger of infection, the others, froze their own or family business, etc. The whole reconstruction of economic life under a state of emergency in Bulgaria leads to changes in both the organization and the content and character of the work

- COVID 19 pandemic leads to significant changes in social life and relations in Bulgarian society. The lives of people (public, professional, family, personal) have actually turned upside down. Every aspect of their daily lives, even the idea of work, communication, leisure, entertainment, etc. has changed. Limited civil rights and freedoms, without which it was not possible to deter and reduce those infected by coronavirus, practically led not only to physical but also to social isolation - and self-confinement within the family and household. At some point, everything became remote - learning, working, shopping. However, partial, incomplete social and business contacts led to difficult communication, alienation and negative mental, emotional and behavioral reactions in some people.

- The institution which suffers the most significant effects and damages from the COVID 19 pandemic is the family. In practice, all responsibility and discipline are transferred to it as a basic social institution with all its functions (reproductive, economic, socialization, spiritual, emotional, etc.). It is assumed that the families who are experiencing most seriously the negative consequences of the pandemic in Bulgaria are predominantly families of: lowstatus groups; vulnerable groups; incomplete nuclear families; with low socio-economic status; low-income families or unemployed; families with young children (in preschool or school age). These should also include single-member households, which similarly experience serious harmful economic and social consequences. As a whole, the quality of life of the Bulgarian family decreased.

\section{State of art}

The COVID-19 is not the first all over the world situation that causes a deep change of the economic and social behavior. The economic and financial crises from $2008-2009$ has the first occasion when the local problem has been fast moved across the world. The COVID-19 has been changing the rules as it covers not just economic and financial impact but also political and social response on the pandemic measures of the Governments.

Having a good understanding of the real, incl. hidden, effects of the economic impact of the COVID-19 is the first step to propose roadmap to fast exit from similar occasions in the future.

Since now, the scientific literature has not given significant proves for the real economic effects of the COVID-19 pandemic measures. There are good researches on the financial and stock-exchange markets [1-3] as well as on employment and staff changes on the micro-level [4-6]. Generally, the researches cover the fast moving sectors that are highly dependent by the COVID-19 restriction measures as: economy lock-down and on-line employment, travel shortage and tourism cut-off, transport routs cut-down and supply chains change, individuals uncertainty and financial market change and etc.

\section{How do the COVID-19 economic effects appear?}

Using the lessons learnt from the Global Financial Crisis (GFC) several researchers report the relationship between financial markets changes and financial stress of individuals and organizations during crises times [3]. The main cause is explained as an unprecedented level of risk rose up that drove investors to suffer significant losses over a very short period [7]. The most important part of the researches is that the one of main channels for fostering 
financial stress is the Economic Policy Uncertainty (EPU) that is driven by the dynamics and change of the industrial production and markets changes. Youssef, Mokni, Ajmi [3] found that the spread of the COVID-19 pandemic caused a high level of dynamic connectedness between international stock markets and increasing economic uncertainty worldwide [3]. This follows a need of better instruments 'not just financial) to avoid panic on the markets at the times of crises.

The crises behavioral change is deeply explained by the theory of FEAR APPEAL [7-8]. The theory is based on the foundation that impulse buying behavior has increased significantly across the world as a result of the fear-inducing COVID-19 phenomenon. In addition, the panic of COVID-19 restrictions has positive and significant impact on impulse buying behavior that transfer long term added value purchasing behavior to significant short term loses individual behavior.

The societal fear is boosted by the level of uncertainty about the true nature of the postcrises' change that will take place. According to the reported changes since now, when once the pandemic is over, there could be a quite feasible economic and social structural change that will move the process of value chains establishment and resp. economic globalization. In addition, COVID-19 crisis still owes a great uncertainty, that allows to conclude that rapid economic recovery cannot be expected. [9]

The current pandemic has impacted over thousands of individuals, covered almost all industry sectors, and caused historical loss to specific industries like the airline industry, oil market, and tourism sector. Thus, there could be found parallels between COVID-19 economic crisis and Global Financial Crisis (GFC) form 2008-2009. Taking into account the above-mentioned features of the COVID-crises, Kolluru et al. [10] expected it to follow a deeper recession and not leave any country unscathed.

On the opposite, Donadelli at al. [11] cannot really capture the real economic effects of COVID-19 that gave an example of epidemic impact of economic recession for the last century. According to them "a rise in the degree of globalization and financial integration, an increase in international labor mobility, and a global and instantaneous diffusion of news will definitely influence the way the economy reacts to epidemic shocks".

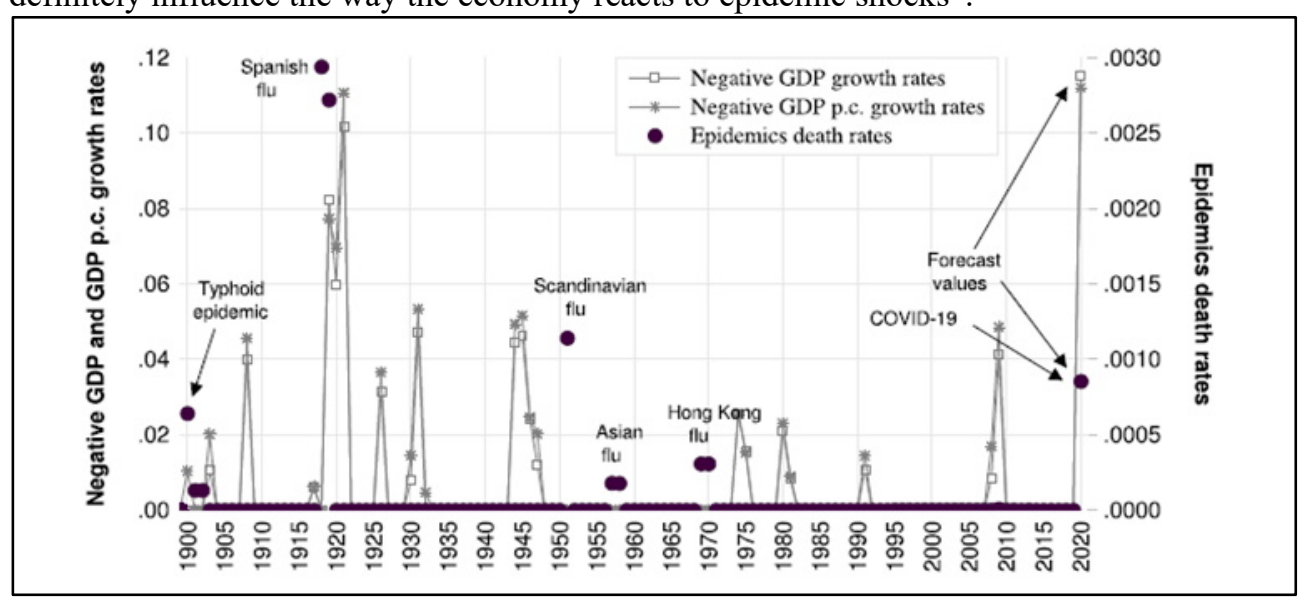

Fig. 1 Epidemics vs. Economic Recessions in England

Source: [11]

Finally, the economic effects of COVID-19 crises spread on decrease of Gross Domestic Product, International trade (import and export values) and change of the Global Value Chains. How deep are changes and if the economic and social transformation is so high as previous crises' depressions could be found by analysis of dependence of total national production (dependent variable) by different independent variables. 


\section{Methodology}

There could be found different approaches to test the economic impact of the COVID-19 pandemic.

As the COVID19 crises causes an increasing economic (and production) risk over time, one of the possible decisions is to use TVP-VAR-based dynamic connectedness approach.

Transforming Youssef, Mokni \& Ajmi [3]

$$
Y_{t}=\Phi_{t} \cdot Y_{t-1}+u_{t}=A_{t} \cdot u_{t}
$$

Where, $Y_{i}-$ is the production of the period i

$\Phi_{t}-$ is the matrix of coefficients, which is supposed to be time-varying

$u_{t}-$ is the vector of the error terms.

$A_{t}=\left(A_{1, t} A_{2, t} \ldots A_{p, t}\right)^{\prime}-$ is the matrix verifying $A_{i, t}=\sum_{k=1}^{p} \Phi_{1, t} \cdot A_{i-k, t}$

Kolluru et al. [10] propose study of economic impact of COVID crises by a qualitative analysis of the (nominal) Gross Domestic Product (GDP). Donadelli et al. [11] use in their analysis the Gross Domestic Product (GDP) but measured by negative GDP growth rates. Additionally, Hayakawa and Mukunoki [12] propose analysis of the impacts of COVID-19 on global value chains by using logged GDP. The construct the next model for analysis:

$$
\begin{gathered}
\text { Trade }_{i j t}=\exp \left\{\alpha_{1} R T A_{i j t}+\alpha_{2} \ln G D P_{i t}+\alpha_{3} \ln G D P_{j t}+\alpha_{4} \operatorname{COVID}_{i t}+\alpha_{5} \operatorname{COVID}_{j t}+\right. \\
\left.\alpha_{6} \operatorname{SCOVID}_{i t}+\delta_{i j}+\delta_{t}\right\} \cdot \epsilon_{i j t}
\end{gathered}
$$

Where, Trade $_{i j t}-$ is export value

$R T A_{i j t}$ - is regional trade agreements

$\operatorname{COVID}_{i t}, \operatorname{COVID}_{j t}$, SCOVID $_{i t}-$ is extend damage of COVID

The real GDP is used by Barthélémy et al. [13] as a part of CRS production function:

$$
Y_{n j}=\left(K_{n j}^{\alpha_{j}} H_{n j}^{1-\alpha_{j}}\right)^{n_{j}} X_{n j}^{1-n_{j}}
$$

Where, $H_{n j}^{1-\alpha_{j}}-$ is aggregate labour input

$K_{n j}^{\alpha_{j}}-$ is aggregate capital input

$X_{n j}^{1-n_{j}}-$ is intermediate input usage

The proposed model is based on dynamic change of the Total factor productivity (TFP) function [15-18] and its transformation of [equation 3] by using dataset of Capital (K), Labor (L) and Intermediate inputs (R).

$$
\Delta Y=Y^{\prime}=\alpha_{1 j} \ln K_{t}+\alpha_{2 j} \ln L_{t}+\alpha_{3 j} \ln R_{t}+\alpha_{0 j}+\varepsilon
$$

In conclusion, the analysis is being done by usage of inputs of 2 independent variables: Import (Imp) and Export (Exp) and their transpose of [equation 1] and [equation 4] to the next:

$$
\Delta Y=Y^{\prime}=\alpha_{1 j} \ln \operatorname{Exp}_{t}-\alpha_{2 j} \ln I m p_{t}+\alpha_{0 j} \operatorname{Crisis}+\varepsilon
$$

Where Crisis - is dummy value for the crises. 
The analysis is based on change of dependence coefficients before COVID 19 crises: $\alpha_{i 1}$ and after COVID crises: $\alpha_{i 2}$

Finally, the role and influence on pandemic social and economic behavior of the COVID19 legal ongoing pandemic measures, have not been fully studied yet. Thus, the real results from the COVID-19 to the society and its economy are unrevealed / unknown, so they are appeared to be HIDDEN EFFECTS / DEFECTS from the current pandemic (COVID-19) crises.

A long run analysis from 2000 to 2019 is proposed to be found the demonstration of economic growth and development opportunities despite the financial turbulence over the crises years.

\section{Data analysis and results}

First prove how deep is the economic impact of the COVID-19 crises since now could be found on the presentation of the Industrial production from 1941 to $2021 \dagger$. (Figure 2)

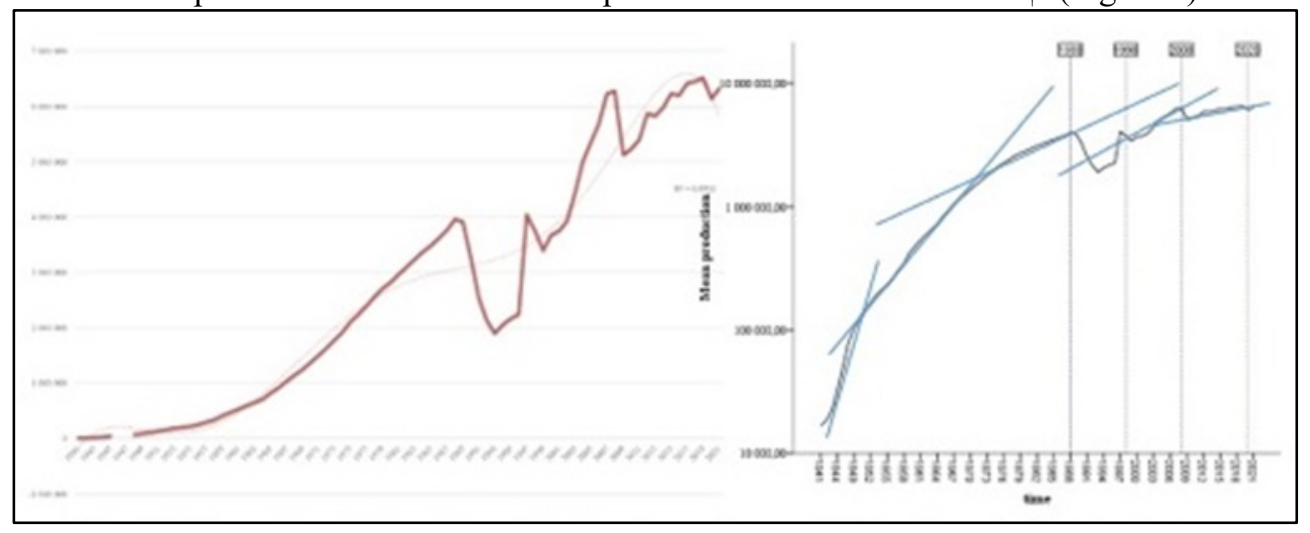

Fig. 2 Mean values (left) and Logarithmic values of change (right) of (nominal) Industrial Production

According to the data (Figure 2) it could be verified the Donadelli at al. [11] that there cannot really capture the economic effects of COVID-19.

Analysis of the exact effect of the crises we need to reduce the dataset. For further analysis, we use data set that covers Bulgarian National Statistic for $2000-2021$ and expresses the main elements of the TFP in [equation 5]: nominal Industrial Production, Industrial Export and Industrial Import of Bulgaria. 3).

The change of the main variables is presented by their logarithmic (ln) function (Figure

Main results from the Figure 3 could be summarized:

- $1999-2008$ (GFC): The production growth is $18.3 \%$, the growth rate of Imports is $46.5 \%$ while the growth of Exports is $36.8 \%$. Production growths slowly with $0.14 \%$ growth rate on monthly basis and $1.7 \%$ growth rate on annual basis. Imports to Bulgaria grew slightly faster $(0.23 \%$ growth rate on a monthly basis and $2.79 \%$ growth rate on an annual basis) than exports of goods from Bulgaria $(0.22 \%$ growth rate on a monthly basis and $2.67 \%$ growth rate on an annual basis)

${ }^{\dagger}$ Expected value for 2021 


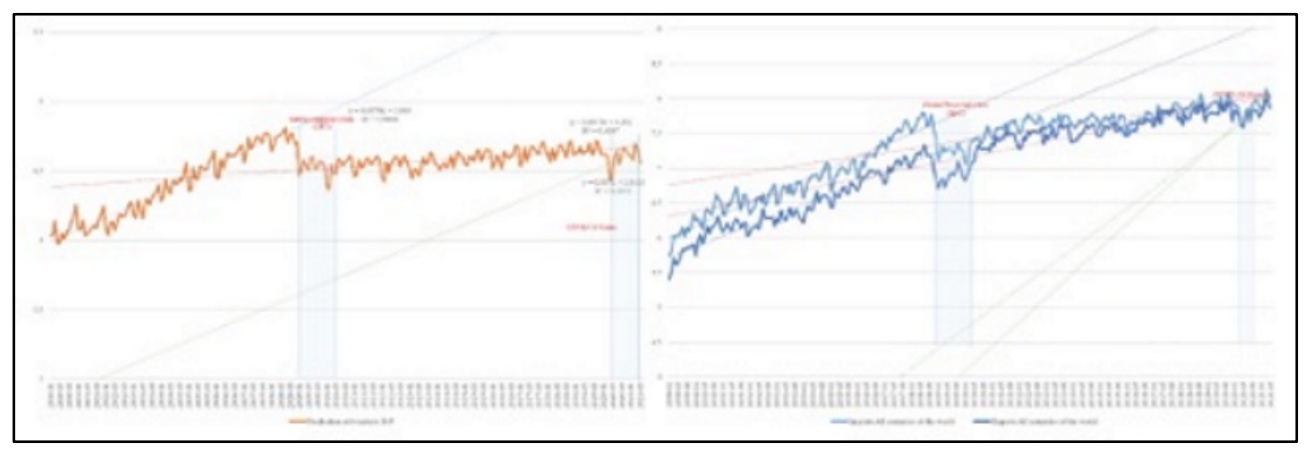

Fig. 3 Logarithmic values of change of Production value (left) and Import and Export (right)

- 2008 (GFC) - 2020 (COVID-19): The production growth is $3.5 \%$ totally and the annual growth rate of production is $0.29 \%$. The rate of increase in imports of goods decreased by $65 \%$ and reached $0.07 \%$ on a monthly basis and $0.84 \%$ on an annual basis, while the rate of increase in exports of goods decreased by $49 \%$ and overtook imports, reaching $0.10 \%$ on a monthly basis and $1.21 \%$ on an annual basis

- 2020 COVID-19) - 2021: The production growth with $2.15 \%$ within monthly growth rate of the growth rate of $0.16 \%$. Imports and exports is growing significantly than the growth in the period 1999-2008, respectively: 6.13 times for imports and 3.73 for exports.

- Major crisis points (GFC 2008-2009) and Covid-19 crisis (April 2020) are reflected in a decrease in the growth rates of imports and exports as follows:

○ Oct.2008: Production falls by $8.5 \%$, imports - by $8.5 \%$ and exports - by $3.6 \%$

○ Apr 2020: Production falls by $6.0 \%$, imports - by $6.7 \%$ and exports - by $3.3 \%$

Analyzing the dataset of the [equation 5], there is found a strong dependence between Production change and Import and Export (Figure 4).

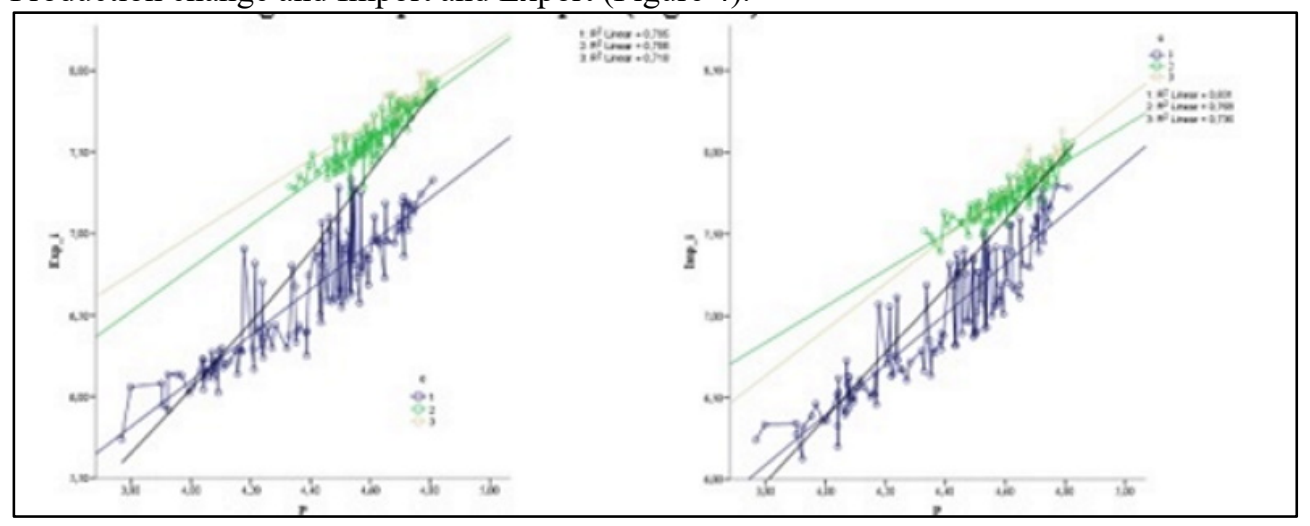

Fig. 4 Regression between logarithmic values of change of Production value and Import (right) and Export (left)

Two main conclusions are done:

- Changes of export in affecting the change of production in the first $(1999-2008)$ and second (2008 - 2020) are equal. For the COVID-19 stage (2020 - 2021) the impact of growth of export to production growth is less. This is a result of "lock-down effect" and loss of international transport routes.

- Change of import in affecting the change of production in the first $(1999-2008)$ and third $(2020-2021)$ are equal too. For the GFC stage $(2008-2020)$ the impact of growth of 
import to production growth is less. This is a result of internal development of new products and process innovations.

Finally, applying the [equation 5] separately to the different stages we find next results (Table 1)

Table 1. Regression coefficient

\begin{tabular}{|c|l|l|l|l|}
\hline Time & $\boldsymbol{\alpha}_{\mathbf{1 j}}$ (Exp) & $\boldsymbol{\alpha}_{\mathbf{2 j}}$ (Imp) & $\boldsymbol{\alpha}_{\mathbf{0} \boldsymbol{j}}$ & $\mathbf{R 2}$ \\
\hline $1999-2021$ & $0.182^{* *}(0.000)$ & $0.889^{*}(0.048)$ & $-0.186^{* *}(0.000)$ & 0.852 \\
\hline $1999-2008$ & $0,381^{* *}(0.000)$ & $0.558^{* *}(0.000)$ & & 0.840 \\
\hline $2008-2020$ & $0.529^{*}(0.000)$ & $0.412^{* *}(0.001)$ & & 0.828 \\
\hline $2020-2021$ & $0.849(0.170)$ & $0.151 \quad(0.977)$ & & 1.000 \\
\hline
\end{tabular}

The results significantly prove that the change of the Export is prior to production growth after any crises. This is a result of inclusion of Bulgarian industry in newer and newer Global added-value chains (GVC).

\section{Conclusions}

Undoubtedly, the COVID-19 crisis is part of the economic life-cycle. As the figure and analysis show, it was time for some changes of the economic and social relations. It was a "economic healing process" and the positives could be much stronger than the negatives.

In addition, post COVID-19 period is defined as "new normality" and "the new normality growth scenario" has to capture structural changes that take place in the economy and in the society. The needed structural changes have to be emerged by technology advances, changing demographics and innovative ways consumers have learned to cope with blurring the work, leisure, and education boundaries [21].

Nevertheless, the COVID-19 crises impacted a lot of individuals and businesses. May be the negative effects of loss jobs and reduced production activities are less than expected because of anti-crises activities all over the world. But to force up the positives of COVID19 crisis the Governments need to re-organize their industrial policy in 3 fields. The needed changes in industrial policy should cover shared resources managements that provide new approach of individuals' economic activity based on mutuality and solidarity to support them including support across supply chains and between producers' cooperation [20].

\subsection{Innovations}

There is need of investments in innovation infrastructure whether any single individual to have opportunity to prove its innovative idea. The innovation infrastructure could cover different start-ups and business incubators. Business incubators could serve as a link between uncertainty and innovations and these innovations will be more adapted to the needs of individuals and there is greater opportunity for launching new successful business models [5]. In addition, economic growth will be push up by social growth via business incubators that could involve in growth trends such as population displacement, violence, youth marginalization, indifference, unemployment, etc.

Furthermore, the business innovation support policy has to be designed to reduce the effect of the "Great Lockdown" on research productivity. As Wang at al. [14] recommend, innovation policy has to be globally oriented, to support break-through innovators, to speed up the process of approving new patents and to target the more innovative individuals. The starting point of the innovation policy could be already reported structural breaks in innovation output across countries and different industries. 


\subsection{Entrepreneurship}

Entrepreneurship is an instrument that prevent job loss in times of economic crises. Thus, there is need to enforce the entrepreneurial policy and to develop entrepreneurial support instruments. Some of the leading economies already enriched their entrepreneurial instruments that provide options countries to explore and to adopt them based on their respective country specific circumstances. Some of them are summarized by Kolluru et al. [10] and cover: introduction of cash for clunkers programs (Germany); launching plans for bank rescue for SMEs' loans and banks lend money to business based on guarantees (Italy and UK). Releasing funds to support small and medium-sized companies (Spain

\subsection{Value chain support}

As the figure 4 show, the Bulgarian industrial production become more and more dependent by development of Global supply chains. So, the successful industrial policy that prevent negative effects of global crises needs to support not just global but domestic value chains as well. In this respect, industrial policy has to support projects for diversification of input sourcing including both domestic and international markets and "dual sourcing" of the same inputs from suppliers in different countries. Such approach should help preventing negative shocks through existing (single or limited number of) value chains. In cases like COVID-19, such support will provide exceptions of lockdown healthy policies for manufacturing and also will reduce the negative effects of lockdowns. [12]

\section{References}

1. D.Y. Aharon, S. Siev, Res. Int. Bus. Finance, 58, 101492 (2021)

2. X. Guo, F. Lu, Y. Wei, Res. Int. Bus. Finance, 58, 101484 (2021)

3. M. Youssef, K. Mokni, A. N. Ajmi, Financ Innov 7, 13 (2021)

4. J. Kettunen, M. Martikainen, G. Voulgaris, J. Bus. Res., 135, 373 - 390 (2021)

5. C. Lin-Lian, C.P. Heredero, J. L. Montes-Botella, Sustainability, 13(12), 6888 (2021)

6. A. Susanty, A. Bakhtiar, F. Prasetya, H. Maher, J.D. Setiawan, C.-C. Chiou, D. Wood, Indicators for Measuring the Impact of COVID-19 on Supply Chain Vulnerability of $S M E s$, in Proceedings of IEEE 8th International Conference on Industrial Engineering and Applications, ICIEA 2021, 23-26 April 2021, virtual, 439 - 443 (2021)

7. L. Eger, L. Komarkova, D. Egerova, D. Gustafsson, J. Retail. Consum. Serv., 61, 102542 (2021)

8. R.R. Ahmed, D. Streimikiene, J-A. Rolle, P.A. Duc, J. Competitiveness, 12(3), 5-27 (2020)

9. V.J. Clemente-Suárez, E. Navarro-Jiménez, L. Moreno-Luna, M.C. Saavedra-Serrano, M. Jimenez, J.A. Simón, J.F. Tornero-Aguilera, Sustainability, 13, 6314 (2021)

10. M. Kolluru, D. Hyams-Ssekasi, K.V.Ch. Madhu Sudhana Rao, ECONOMICS, 9(1), 85-105 (2021)

11. M. Donadelli, L. Ferranna, I. Gufler, A. Paradiso, Struct. Change Econ. Dyn, 57, 214224 (2021)

12. K. Hayakawa, H. Mukunoki, Dev. Econ, 59(2), 154-177 (2021)

* The same instrument was used in Bulgaria 
13. B. Bonadio, Zhen Huo, A.A. Levchenko, N. Pandalai-Nayar, NBER Working Papers 27224, (2020)

14. L. Wang, M. Zhang, T. Verousis, Int. Rev. Financ. Anal, 75, 101729 (2021)

15. N. Bloom, P. Bunn, P. Mizen, P. Smietanka, G. Thwaites, NBER Working Paper 28233, (2021)

16. N. Sterev, D. Kopeva, D. Blagoev, IJBESAR, 10(1), 39-54 (2017)

17. N. Sterev, D. Blagoev, I. Gatovski, Economic Alternatives, 1, 19 -32 (2014)

18. W. Feng, L. Jun, G. Liang, L. Zhihui, X. Deng, Acta Geographica Sinica, 76(4), 1034$1048(2021)$

19. D. Zhang, M. Hu, Q. Ji, Finance Res Lett, 36, 101528 (2020)

20. M. Leach, H. MacGregor, I. Scoones, A. Wilkinson, World Development, 138(7), 105233 (2021)

21. R. Capello, A. Caragliu, J Regional Sci., 1- 18 (2021) 